\title{
Editorial: Physical Review $D$ expands coverage of astrophysics and astronomy
}

Physical Review D is a leading journal in high-energy physics, gravitation, and cosmology, and it already has a strong presence in astrophysics, covering particle astrophysics, gravitational waves, cosmic-ray physics, and dark matter. For example, the journal has published leading papers by the LIGO Collaboration, from the Dark Energy Survey, and on dark matter searches by XENON and other experiments. Based on these strengths, the Editorial Team is building a welcoming home for rapidly growing subfields of astrophysics and astronomy, such as multimessenger astrophysics, high-energy neutrino astrophysics, and galactic and extragalactic astronomy. These subfields all have substantial overlap with the scientific scope of PRD.

We are therefore delighted to announce that astrophysicist Enrico Ramirez-Ruiz has been appointed as a new Associate Editor of PRD, as of October 1, 2021. Ramirez-Ruiz is a Professor and the Vera Rubin Presidential Chair of Astronomy and Astrophysics at the University of California, Santa Cruz. He focuses on developing the conceptual framework needed to understand the violent nature of the Universe with an emphasis on stellar explosions, gamma-ray bursts, and accretion phenomena. He is particularly interested in understanding the physical processes that govern accretion onto relativistic objects such as black holes and neutron stars. He uses computer simulations to explore transient phenomena such as mergers and disruptions of stars, especially those involving compact objects. Ramirez-Ruiz's work has been recognized with a Packard Fellowship, a U.S. National Science Foundation CAREER Award, the Edward A. Bouchet Award from the American Physical Society (APS), and the HEAD Mid-Career Prize from the American Astronomical Society.

Ramirez-Ruiz has also worked vigorously to support the promotion and retention of women and people from underrepresented groups in astronomy and related fields. He established and directs the Lamat program, which gives undergraduate students, mostly from historically marginalized groups, the opportunity to work with UCSC faculty and graduate students on research projects using some of the world's most advanced computers.

Ramirez-Ruiz's impressive achievements and scientific ties to the emerging subfields of astrophysics and astronomy will be instrumental in developing strong connections between researchers in these subfields and PRD, which in turn should raise the journal's overall visibility and high profile.

As a further important step in these directions, with Ramirez-Ruiz's help and support, we have appointed three new Editorial Board Members: Manuela Campanelli, Daniel Kasen, and Samaya Nissanke. They started their respective three-year appointments on January 1, 2022.

Manuela Campanelli is a Distinguished Professor in the School of Mathematical Sciences at the Rochester Institute of Technology, where she serves as the Director of the Center for Computational Relativity and Gravitation. Her renowned work focuses on the astrophysics of merging black holes and neutron stars, which are powerful sources of gravitational waves, electromagnetic radiation, and relativistic jets. She is leading state-of-the-art simulations of binary neutron stars that aim at providing an understanding of multimessenger astrophysics observations of these systems. She is the Theoretical and Computational Astrophysics Networks (TCAN) NASA director and a Fellow of APS.

Daniel Kasen is a Professor in the Department of Astronomy and Astrophysics at the University of California, Berkeley. He is a renowned theoretical and computational astrophysicist, focusing on supernovae and other energetic transients. He is a recipient of the Rossi Prize from the American Astronomical Society for his innovative works on predicting the electromagnetic signals from neutron star mergers.

Samaya Nissanke is an Associate Professor and the spokesperson for the Gravitation and AstroParticle Physics Center of Excellence (GRAPPA) at the University of Amsterdam. Her work on gravitational-wave astrophysics has played a founding role in the emerging field of multimessenger astronomy. She is member of the Virgo Collaboration, co-chairing the Virgo O5+ committee. She is the University of Amsterdam representative for the BlackGEM telescope. As part of the Virgo and LIGO Collaborations, she was awarded the Special 
Breakthrough Prize in Fundamental Physics (2016), and won the 2020 New Horizons in Physics Prize from the Breakthrough Prize Foundation.

Please join us in welcoming this exciting cohort of experts to the PRD family.

(Q) Published 24 January 2022

Mirjam Cvetič

Lead Editor

Urs M. Heller

Managing Editor

American Physical Society

DOI: 10.1103/PhysRevD.105.020001 